\title{
Harnack inequality for parabolic Lichnerowicz equations on complete noncompact Riemannian manifolds
}

\section{Liang Zhao*}

\section{"Correspondence: \\ zhaozongliang09@163.com \\ Department of Mathematics, \\ Nanjing University of Aeronautics \\ and Astronautics, Nanjing, 210016, \\ P.R. China}

\begin{abstract}
In this paper, we study the gradient estimates for positive solutions to the following parabolic Lichnerowicz equations

$$
\frac{\partial u}{\partial t}=\Delta u+h u(x, t)+A u^{p}(x, t)+B u^{-q}(x, t)
$$

on complete noncompact Riemannian manifolds, where $h, p, q, A, B$ are real constants and $p>1, q>0$.

MSC: Primary 58J05; secondary 58J35

Keywords: Lichnerowicz equation; positive solutions; Harnack inequality
\end{abstract}

\section{Introduction}

Let $M$ be an $n$-dimensional complete noncompact Riemannian manifold. In this paper, we study the following nonlinear parabolic equation

$$
u_{t}(x, t)=\Delta u(x, t)+h u(x, t)+A u^{p}(x, t)+B u^{-q}(x, t),
$$

where $h, p, q, A, B$ are real constants and $p>1, q>0$.

Gradient estimates play an important role in the study of PDE, especially the Laplace equation and heat equation. Li [1] derived the gradient estimates and Harnack inequalities for positive solutions of nonlinear equations $\left(\triangle-\frac{\partial}{\partial t}\right) u(x, t)+h(x, t) u^{\alpha}(x, t)=0$ and $A u+$ $b \nabla u+h u^{\alpha}=0$ on Riemannian manifolds. The author in [1] also obtained a theorem of Liouville-type for positive solutions of the nonlinear elliptic equation. Later, Yang [2] gave the gradient estimates for the solution to the elliptic equation with singular nonlinearity

$$
\triangle u+c u^{-\alpha}=0
$$

where $\alpha>0, c$ are two real constants. More precisely, the author [2] obtained the following result.

Theorem 1.1 (Yang [2]) Let $M$ be a noncompact complete Riemannian manifold of dimension $n$ without boundary. Let $B_{p}(2 R)$ be a geodesic ball of radius $2 R$ around $p \in M$. We denote $-K(2 R)$, with $K(2 R) \geq 0$, to be a lower bound of the Ricci curvature on $B_{p}(2 R)$, 
i.e., $\operatorname{Ric}(\xi, \xi) \geq-K(2 R)|\xi|^{2}$ for all tangent field $\xi$ on $B_{p}(2 R)$. Suppose that $u(x)$ is a positive smooth solution of the equation (1.2) with $\alpha>0, c$ being two real constants. Then we have:

(i) If $c>0$, then $u(x)$ satisfies the estimate

$$
\frac{|\nabla u|^{2}}{u^{2}}+c u^{-(\alpha+1)} \leq \frac{n(2 n+1) \epsilon^{2}}{R^{2}}+\frac{n(n-1) \epsilon^{2}}{R} \sqrt{K(2 R)}+\frac{n v}{R^{2}}+2 n K(2 R)
$$

on $B_{p}(R)$, where $\epsilon>0$ and $v>0$ are some universal constants independent of geometry of $M$.

(ii) If $c<0$, then $u(x)$ satisfies the estimate

$$
\begin{aligned}
\frac{|\nabla u|^{2}}{u^{2}}+c u^{-(\alpha+1)} \leq & (n(\alpha+1)(\alpha+2)+\sqrt{n}(\alpha+1))|c|\left(\inf _{B_{p}(2 R)} u\right)^{-\alpha-1} \\
& +\frac{n v}{R^{2}}+\left(2 n+\frac{\sqrt{n}}{\alpha+1}\right) K(2 R) \\
& +\frac{n \epsilon^{2}}{R^{2}}(2 n+1)+\frac{\sqrt{n}}{2(\alpha+1)}+(n-1) \sqrt{K(2 R) R}
\end{aligned}
$$

on $B_{p}(R)$, where $\epsilon>0$ and $v>0$ are some universal constants independent of geometry of $M$.

For some interesting gradient estimates in this direction, we can refer to [3-7]. Recently, Song and Zhao [8] studied a generalized elliptic Lichnerowicz equation

$$
\Delta u(x)+h(x) u(x)=A(x) u^{p}(x)+\frac{B(x)}{u^{q}(x)}
$$

on compact manifold $(M, g)$. The authors in [8] got the local gradient estimate for the positive solutions of (1.3). Moreover, they considered the following parabolic Lichnerowicz equation

$$
u_{t}(x, t)+\Delta u(x, t)+h(x) u(x, t)=A(x) u^{p}(x, t)+B(x) u^{-q}(x, t)
$$

on manifold $(M, g)$ and obtained the Harnack differential inequality.

Theorem 1.2 (Song and Zhao [8]) Let $M$ be a compact Riemannian manifold without boundary, $\operatorname{Ric}(M) \geq 0$. Let $c(t) \in C^{1}(0, \infty)$. Assume that $u(x, t)$ is any positive solution of (1.4) on $M$ with $A(x) \equiv A, B(x) \equiv B$, and $h(x) \equiv h$. Denote $\varphi=\ln u$, suppose that $A \leq 0$, $B \geq 0, c(t) \geq 0, c^{\prime}(t) \geq 0$. If $|\nabla \varphi|^{2}-\frac{1}{p} \varphi_{t}+\frac{1}{p} \widetilde{H}-c(t) \leq 0$ with $\widetilde{H}=A e^{(p-1) \varphi}+\frac{B}{e^{(q+1) \varphi}}-h a t$ $t=0$, then we have

$$
|\nabla \varphi|^{2}-\frac{1}{p} \varphi_{t}+\frac{1}{p} \widetilde{H}-c(t) \leq 0
$$

While the author considered the gradient estimates on compact Riemannian manifolds in Theorem 1.2, it is natural to study this problem on complete noncompact manifolds. Motivated by the work above, we present our main results as follows.

Theorem 1.3 Let $(M, g)$ be a complete noncompact $n$-dimensional Riemannian manifold with Ricci tensor bounded from below by the constant $-K=:-K(2 R)$, where $R>0$ and 
$K(2 R)>0$ in the metric ball $B_{2 R}(p)$ around $p \in M$. Assume that $u$ is a positive solution of $(1.1)$ with $u \leq M_{1}$ for all $(x, t) \in B_{R}(p) \times(0,+\infty)$. Then

(1) if $A \leq 0, B \geq 0$, we have

$$
\begin{aligned}
& \beta \frac{|\nabla u|^{2}}{u^{2}}+h+A u^{p-1}+B u^{-(q+1)}-\frac{u_{t}}{u} \\
& \quad \leq \frac{n}{2(1-\delta) \beta}\left(\frac{n c_{1}^{2}}{4 \delta \beta(1-\beta) R^{2}}-\frac{M_{1}^{p-1} A(p-1)(p-\beta)}{4(1-\beta)^{2}}+H+\frac{1}{t}\right)
\end{aligned}
$$

(2) if $A>0, B>0$, we get

$$
\begin{aligned}
& \beta \frac{|\nabla u|^{2}}{u^{2}}+h+A u^{p-1}+B u^{-(q+1)}-\frac{u_{t}}{u} \\
& \leq \frac{n}{2(1-\delta) \beta}\left(\frac{n c_{1}^{2}}{4 \delta \beta(1-\beta) R^{2}}+M_{1}^{p-1} A(p-1)+H+\frac{1}{t}\right),
\end{aligned}
$$

where $H=\frac{(n-1)(1+\sqrt{K} R) c+c_{2}+2 c_{1}^{2}}{R^{2}}, c, c_{1}, c_{2}, \delta$ are positive constants with $0<\delta<1$ and $\beta=e^{-2 K t}$.

Let $R \rightarrow \infty$, we can get the following global gradient estimates for the nonlinear parabolic equation (1.1).

Corollary 1.4 Let $(M, g)$ be a complete noncompact $n$-dimensional Riemannian manifold with Ricci tensor bounded from below by the constant $-K=:-K(M)$, where $K>0$. Assume that $u$ is a positive solution of (1.1) with $u \leq M_{1}$ for all $(x, t) \in M \times(0,+\infty)$. Then

(1) if $A \leq 0, B \geq 0$, we have

$$
\beta \frac{|\nabla u|^{2}}{u^{2}}+h+A u^{p-1}+B u^{-(q+1)}-\frac{u_{t}}{u} \leq \frac{n}{2(1-\delta) \beta}\left(-\frac{M_{1}^{p-1} A(p-1)(p-\beta)}{4(1-\beta)^{2}}+\frac{1}{t}\right)
$$

(2) if $A>0, B>0$, we get

$$
\beta \frac{|\nabla u|^{2}}{u^{2}}+h+A u^{p-1}+B u^{-(q+1)}-\frac{u_{t}}{u} \leq \frac{n}{2(1-\delta) \beta}\left(M_{1}^{p-1} A(p-1)+\frac{1}{t}\right),
$$

$\delta$ are positive constants with $0<\delta<1$ and $\beta=e^{-2 K t}$.

Let $\delta \rightarrow 0, A=0$ in Corollary 1.4, we get a Li-Yau-type gradient estimate.

Corollary 1.5 Let $(M, g)$ be a complete noncompact $n$-dimensional Riemannian manifold with $\operatorname{Ric}(M) \geq 0$. Assume that $u(x, t)$ is a positive solution to the equation

$$
\frac{\partial u}{\partial t}=\Delta u+h u(x, t)+B u^{-q}(x, t)
$$

on complete noncompact manifolds, where $h, q, B$ are real constants and $q>0$. Then we have

$$
\frac{|\nabla u|^{2}}{u^{2}}+h+B u^{-(q+1)}-\frac{u_{t}}{u} \leq \frac{n}{2 t} .
$$

As an application, we have the following Harnack inequality. 
Theorem 1.6 Let $(M, g)$ be a complete noncompact $n$-dimensional Riemannian manifold with $\operatorname{Ric}(M) \geq 0$. Assume that $u(x, t)$ is a positive solution to the equation

$$
\frac{\partial u}{\partial t}=\Delta u+h u(x, t)+B u^{-q}(x, t)
$$

on complete noncompact manifolds, where $h, q, B$ are real constants and $q>0, B>0$. Then for any points $\left(x_{1}, t_{1}\right)$ and $\left(x_{2}, t_{2}\right)$ on $M \times[0,+\infty)$ with $0<t_{1}<t_{2}$, we have the following Harnack inequality:

$$
u\left(x_{1}, t_{1}\right) \leq u\left(x_{2}, t_{2}\right)\left(\frac{t_{2}}{t_{1}}\right)^{\frac{n}{2}} e^{\phi\left(x_{1}, x_{2}, t_{1}, t_{2}\right)+D},
$$

where $\phi\left(x_{1}, x_{2}, t_{1}, t_{2}\right)=\frac{d^{2}\left(x_{1}, x_{2}\right)}{t_{2}-t_{1}}, D=h\left(t_{1}-t_{2}\right)$.

\section{Proof of Theorem 1.3}

Assume that $u$ is a positive solution to (1.1). Set $w=\ln u$, then $w$ satisfies the equation

$$
w_{t}=\Delta w+|\nabla w|^{2}+h+A e^{(p-1) w}+B e^{-w(q+1)} .
$$

Lemma 2.1 Let $(M, g)$ be a complete noncompact $n$-dimensional Riemannian manifold with Ricci curvature bounded from below by the constant $-K=:-K(2 R)$, where $R>0$ and $K>0$ in the metric ball $B_{2 R}(p)$ around $p \in M$. Let $w$ be a positive solution of $(2.1)$, then

$$
\begin{aligned}
\left(\Delta-\frac{\partial}{\partial t}\right) F \geq & -2 \nabla w \cdot \nabla F+t\left\{\frac{2 \beta}{n}\left((\beta-1)|\nabla w|^{2}-\frac{F}{t}\right)^{2}\right. \\
& \left.+A(p-\beta)(p-1) e^{(p-1) w}|\nabla w|^{2}+B(q+1)(q+\beta) e^{-w(q+1)}|\nabla w|^{2}\right\} \\
& +\left[B(q+1) e^{-(q+1) w}-A(p-1) e^{(p-1) w}\right] F-\frac{F}{t},
\end{aligned}
$$

where

$$
F=t\left(\beta|\nabla w|^{2}+h+A e^{(p-1) w}+B e^{-(q+1) w}-w_{t}\right),
$$

and $\beta=e^{-2 K t}$.

Proof Define

$$
F=t\left(\beta|\nabla w|^{2}+h+A e^{(p-1) w}+B e^{-(q+1) w}-w_{t}\right),
$$

where $\beta=e^{-2 K t}$. By the Bochner formula, we have

$$
\Delta|\nabla w|^{2} \geq \frac{2}{n}|\triangle w|^{2}+2 \nabla w \nabla(\Delta w)-2 K|\nabla w|^{2} .
$$

By a direct computation, we have

$$
\Delta w_{t}=(\Delta w)_{t}=-2 \nabla w \nabla w_{t}-A(p-1) e^{(p-1) w} w_{t}+B(q+1) e^{-(q+1) w} w_{t}+w_{t t}
$$


and

$$
\begin{aligned}
\Delta w & =-|\nabla w|^{2}-h-A e^{(p-1) w}-B e^{-(q+1) w}+w_{t} \\
& =\left(1-\frac{1}{\beta}\right)\left(-h-A e^{(p-1) w}-B e^{-(q+1) w}+w_{t}\right)-\frac{F}{\beta t} \\
& =(\beta-1)|\nabla w|^{2}-\frac{F}{t},
\end{aligned}
$$

and we know

$$
\begin{aligned}
\Delta F= & t\left\{\beta \Delta|\nabla w|^{2}+A\left[(p-1)^{2} e^{(p-1) w}|\nabla w|^{2}+(p-1) e^{(p-1) w} \Delta w\right]\right. \\
& \left.+B\left[(q+1)^{2} e^{-(q+1) w}|\nabla w|^{2}-(q+1) e^{-(q+1) w} \Delta w\right]-\Delta w_{t}\right\}
\end{aligned}
$$

Therefore, by equalities (2.2) and (2.3), we obtain

$$
\begin{aligned}
\beta \Delta|\nabla w|^{2} \geq & \frac{2 \beta}{n}\left((\beta-1)|\nabla w|^{2}-\frac{F}{t}\right)^{2}+2 \beta \nabla w \nabla(\Delta w)-2 \beta K|\nabla w|^{2} \\
= & \frac{2 \beta}{n}\left((\beta-1)|\nabla w|^{2}-\frac{F}{t}\right)^{2} \\
& +2 \beta \nabla w \nabla\left[\left(1-\frac{1}{\beta}\right)\left(-h-A e^{(p-1) w}-B e^{-(q+1) w}+w_{t}\right)-\frac{F}{\beta t}\right] \\
& -2 \beta K|\nabla w|^{2} \\
= & \frac{2 \beta}{n}\left((\beta-1)|\nabla w|^{2}-\frac{F}{t}\right)^{2}-2 A(\beta-1)(p-1) e^{(p-1) w}|\nabla w|^{2} \\
& +2 B(\beta-1)(q+1) e^{-(q+1) w}|\nabla w|^{2}+2(\beta-1) \nabla w \nabla w_{t} \\
& -\frac{2}{t} \nabla w \nabla F-2 \beta K|\nabla w|^{2} .
\end{aligned}
$$

This implies that,

$$
\begin{aligned}
\Delta F \geq & t \frac{2 \beta}{n}\left((\beta-1)|\nabla w|^{2}-\frac{F}{t}\right)^{2}-2 A(\beta-1)(p-1) e^{(p-1) w}|\nabla w|^{2} \\
& +2 B(\beta-1)(q+1) e^{-(q+1) w}|\nabla w|^{2}+2(\beta-1) \nabla w \nabla w_{t} \\
& -\frac{2}{t} \nabla w \nabla F-2 \beta K|\nabla w|^{2}+A(p-1)^{2} e^{(p-1) w}|\nabla w|^{2} \\
& +A(p-1) e^{(p-1) w}\left[(\beta-1)|\nabla w|^{2}-\frac{F}{t}\right]+B(q+1)^{2} e^{-(q+1) w}|\nabla w|^{2} \\
& -B(q+1) e^{-(q+1) w}\left[(\beta-1)|\nabla w|^{2}-\frac{F}{t}\right]+2 \nabla w \nabla w_{t}+A(p-1) e^{(p-1) w} w_{t} \\
& \left.-B(q+1) e^{-(q+1) w} w_{t}-w_{t t}\right\}
\end{aligned}
$$

and

$$
F_{t}=\frac{F}{t}+t\left(2 \beta \nabla w \nabla w_{t}+A(p-1) e^{(p-1) w} w_{t}-B(q+1) e^{-(q+1) w} w_{t}-w_{t t}-2 K \beta|\nabla w|^{2}\right)
$$


Therefore, it follows that

$$
\begin{aligned}
\left(\Delta-\frac{\partial}{\partial t}\right) F \geq & -2 \nabla w \cdot \nabla F+t\left\{\frac{2 \beta}{n}\left((\beta-1)|\nabla w|^{2}-\frac{F}{t}\right)^{2}\right. \\
& \left.+A(p-\beta)(p-1) e^{(p-1) w}|\nabla w|^{2}+B(q+1)(q+\beta) e^{-w(q+1)}|\nabla w|^{2}\right\} \\
& +\left[B(q+1) e^{-(q+1) w}-A(p-1) e^{(p-1) w}\right] F-\frac{F}{t},
\end{aligned}
$$

which completes the proof of Lemma 2.1.

We take a $C^{2}$ cut-off function $\tilde{\varphi}$ defined on $[0, \infty)$ such that $\tilde{\varphi}(r)=1$ for $r \in[0,1], \tilde{\varphi}(r)=0$ for $r \in[2, \infty)$, and $0 \leq \tilde{\varphi}(r) \leq 1$. Furthermore, $\tilde{\varphi}$ satisfies

$$
\frac{\tilde{\varphi}^{\prime}(r)}{\tilde{\varphi}^{\frac{1}{2}}(r)} \geq-c_{1}
$$

and

$$
\tilde{\varphi}^{\prime \prime}(r) \geq-c_{2}
$$

for some absolute constants $c_{1}, c_{2}>0$. Denote by $r(x)$ the distance between $x$ and $p$ in $M$.

Set

$$
\varphi(x)=\tilde{\varphi}\left(\frac{r(x)}{R}\right)
$$

Using an argument of Cheng and Yau [9], we can assume that $\varphi(x) \in C^{2}(M)$ with support in $B_{p}(2 R)$. Direct calculation shows that on $B_{p}(2 R)$

$$
\frac{|\nabla \varphi|^{2}}{\varphi} \leq \frac{c_{1}^{2}}{R^{2}}
$$

By the Laplacian comparison theorem in [10],

$$
\triangle \varphi \geq-\frac{(n-1)(1+\sqrt{K} R) c_{1}^{2}+c_{2}}{R^{2}} \quad\left(c_{1} \geq 1\right) .
$$

In inequality (2.5), if $c_{1}<1$, then $\triangle \varphi$ can be controlled by $-\frac{(n-1)(1+\sqrt{K} R) c_{1}+c_{2}}{R^{2}}$, so in any case, $\triangle \varphi \geq-\frac{(n-1)(1+\sqrt{K} R) c+c_{2}}{R^{2}}$, where $c$ is some positive constant.

For $T \geq 0$, let $(x, s)$ be a point in $B_{2 R}(p) \times[0, T]$, at which $\varphi F$ attains its maximum value $P$, and we assume that $P$ is positive (otherwise the proof is trivial). At the point $(x, s)$, we have

$$
\nabla(\varphi F)=0, \quad \triangle(\varphi F) \leq 0, F_{t} \geq 0 .
$$

It follows that

$$
\varphi \triangle F+F \triangle \varphi-2 F \varphi^{-1}|\nabla \varphi|^{2} \leq 0 .
$$


This inequality, together with inequalities (2.4) and (2.5), yields

$$
\varphi \triangle F \leq H F,
$$

where

$$
H=\frac{((n-1)(1+\sqrt{K} R)) c+c_{2}+2 c_{1}^{2}}{R^{2}} .
$$

At $(x, s)$, by Lemma 2.1 , we have

$$
\begin{aligned}
\varphi \triangle F \geq & -2 \varphi \nabla w \nabla F+s \varphi\left\{\frac{2 \beta}{n}\left((\beta-1)|\nabla w|^{2}-\frac{F}{s}\right)^{2}\right. \\
& \left.+A(p-\beta)(p-1) e^{(p-1) w}|\nabla w|^{2}+B(q+1)(q+\beta) e^{-w(q+1)}|\nabla w|^{2}\right\} \\
& +\varphi\left[B(q+1) e^{-(q+1) w}-A(p-1) e^{(p-1) w}\right] F-\varphi \frac{F}{s},
\end{aligned}
$$

it follows that

$$
\begin{aligned}
\frac{2 s \varphi \beta}{n}\left((\beta-1)|\nabla w|^{2}-\frac{F}{s}\right)^{2} \leq & \frac{2 c_{1}}{R} \varphi^{\frac{1}{2}} F|\nabla w|+H F-A(p-\beta)(p-1) e^{(p-1) w}|\nabla w|^{2} s \varphi \\
& -B(q+1)(q+\beta) e^{-w(q+1)}|\nabla w|^{2} s \varphi \\
& -\left[B(q+1) e^{-(q+1) w}-A(p-1) e^{(p-1) w}\right] \varphi F+\varphi \frac{F}{s}
\end{aligned}
$$

here we used

$$
-2 \varphi \nabla w \nabla F=2 F \nabla w \nabla \varphi \geq-2 F|\nabla w||\nabla \varphi| \geq-\frac{2 c_{1}}{R} \varphi^{\frac{1}{2}} F|\nabla w| .
$$

Following Davies [11] (see also Negrin [12]), we set

$$
\mu=\frac{|\nabla w|^{2}}{F} .
$$

Then we have

$$
\begin{aligned}
\frac{2 \varphi \beta((\beta-1) s \mu-1)^{2} F^{2}}{n s} \leq & \frac{2 c_{1}}{R} \varphi^{\frac{1}{2}} \mu^{\frac{1}{2}} F^{\frac{3}{2}}+H F-A(p-\beta)(p-1) e^{(p-1) w} s \varphi \mu F \\
& -B(q+1)(q+\beta) e^{-w(q+1)} s \mu \varphi F \\
& -\left[B(q+1) e^{-(q+1) w}-A(p-1) e^{(p-1) w}\right] \varphi F+\varphi \frac{F}{s} .
\end{aligned}
$$

Next, we consider the following two cases:

(1) if $A \leq 0, B \geq 0$, then we have

$$
\frac{2 \varphi \beta((\beta-1) s \mu-1)^{2} F^{2}}{n s} \leq \frac{2 c_{1}}{R} \varphi^{\frac{1}{2}} \mu^{\frac{1}{2}} F^{\frac{3}{2}}+H F-M_{1}^{p-1} A(p-1)(p-\beta) \mu s \varphi F+\frac{\varphi F}{s},
$$


multiplying both sides of the inequality above by $s \varphi$, we have

$$
\begin{aligned}
\frac{2 \beta((\beta-1) s \mu-1)^{2}}{n}(\varphi F)^{2} \leq & \frac{2 c_{1}}{R} s \mu^{\frac{1}{2}}(\varphi F)^{\frac{3}{2}}+H s \varphi F-M_{1}^{p-1} A(p-1)(p-\beta) \mu s^{2} \varphi F+\varphi F \\
\leq & \frac{2 \delta \beta((\beta-1) s \mu-1)^{2}}{n}(\varphi F)^{2}+\frac{n c_{1}^{2} s^{2} \mu}{2 \delta \beta((\beta-1) s \mu-1)^{2} R^{2}} \varphi F \\
& +H s \varphi F-M_{1}^{p-1} A(p-1)(p-\beta) \mu s^{2} \varphi F+\varphi F .
\end{aligned}
$$

So, it follows that

$$
\begin{aligned}
P \leq & \frac{n}{2(1-\delta) \beta((\beta-1) s \mu-1)^{2}} \\
& \times\left(\frac{n c_{1}^{2} s^{2} \mu}{2 \delta \beta((\beta-1) s \mu-1)^{2} R^{2}}-\frac{M_{1}^{p-1} A(p-1)(p-\beta) \mu s^{2}}{2(1-\beta)}+H s+1\right) .
\end{aligned}
$$

Since

$$
((\beta-1) s \mu-1)^{2} \geq 2(1-\beta) s \mu+1 \geq 2(1-\beta) s \mu,
$$

we get

$$
P \leq \frac{n}{2(1-\delta) \beta}\left(\frac{n c_{1}^{2} s}{4 \delta \beta(1-\beta) R^{2}}-\frac{M_{1}^{p-1} A(p-1)(p-\beta) s}{4(1-\beta)^{2}}+H s+1\right) .
$$

Now, (1) of Theorem 1.3 can be easily deduced from the inequality above;

(2) if $A>0, B>0$, then we have

$$
\frac{2 \varphi \beta((\beta-1) s \mu-1)^{2} F^{2}}{n s} \leq \frac{2 c_{1}}{R} \varphi^{\frac{1}{2}} \mu^{\frac{1}{2}} F^{\frac{3}{2}}+M_{1}^{p-1} A(p-1) \varphi F+H F+\frac{\varphi F}{s},
$$

multiplying both sides of the inequality above by $s \varphi$, we have

$$
\begin{aligned}
\frac{2 \beta((\beta-1) s \mu-1)^{2}}{n}(\varphi F)^{2} \leq & \frac{2 c_{1}}{R} s \mu^{\frac{1}{2}}(\varphi F)^{\frac{3}{2}}+M_{1}^{p-1} A(p-1) \varphi F s+H s \varphi F+\varphi F \\
\leq & \frac{2 \delta \beta((\beta-1) s \mu-1)^{2}}{n}(\varphi F)^{2}+\frac{n c_{1}^{2} s^{2} \mu}{2 \delta \beta((\beta-1) s \mu-1)^{2} R^{2}} \varphi F \\
& +M_{1}^{p-1} A(p-1) \varphi F s+H s \varphi F+\varphi F .
\end{aligned}
$$

So, it follows that

$$
P \leq \frac{n}{2(1-\delta) \beta}\left(\frac{n c_{1}^{2} s}{4 \delta \beta(1-\beta) R^{2}}+M_{1}^{p-1} A(p-1) s+H s+1\right) .
$$

Similarly, we can obtain (2) of Theorem 1.3.

Proof of Theorem 1.6 For any points $\left(x_{1}, t_{1}\right)$ and $\left(x_{2}, t_{2}\right)$ on $M \times[0,+\infty)$ with $0<t_{1}<t_{2}$, we take a curve $\gamma(t)$ parameterized with $\gamma\left(t_{1}\right)=x_{1}$ and $\gamma\left(t_{2}\right)=x_{2}$. One gets from Corollary 1.5 
that

$$
\begin{aligned}
\log u\left(x_{2}, t_{2}\right)-\log u\left(x_{1}, t_{1}\right) & =\int_{t_{1}}^{t_{2}}\left((\log u)_{t}+\langle\nabla \log u, \dot{\gamma}\rangle\right) d t \\
& \geq \int_{t_{1}}^{t_{2}}\left(|\nabla \log u|^{2}-\frac{n}{2 t}+h+B u^{-(q+1)}-|\nabla \log u||\dot{\gamma}|\right) d t \\
& \geq-\int_{t_{1}}^{t_{2}}\left(\frac{1}{4}|\dot{\gamma}|^{2}+\frac{n}{2 t}-h\right) d t \\
& =-\left(\int_{t_{1}}^{t_{2}} \frac{1}{4}|\dot{\gamma}|^{2} d t+\log \left(\frac{t_{2}}{t_{1}}\right)^{\frac{n}{2}}+h\left(t_{1}-t_{2}\right)\right),
\end{aligned}
$$

which means that

$$
\log \frac{u\left(x_{1}, t_{1}\right)}{u\left(x_{2}, t_{2}\right)} \leq \int_{t_{1}}^{t_{2}} \frac{1}{4}|\dot{\gamma}|^{2} d t+\log \left(\frac{t_{2}}{t_{1}}\right)^{\frac{n}{2}}+h\left(t_{1}-t_{2}\right) .
$$

Therefore,

$$
u\left(x_{1}, t_{1}\right) \leq u\left(x_{2}, t_{2}\right)\left(\frac{t_{2}}{t_{1}}\right)^{\frac{n}{2}} e^{\phi\left(x_{1}, x_{2}, t_{1}, t_{2}\right)+D},
$$

where $\phi\left(x_{1}, x_{2}, t_{1}, t_{2}\right)=\frac{d^{2}\left(x_{1}, x_{2}\right)}{t_{2}-t_{1}}, D=h\left(t_{1}-t_{2}\right)$.

\section{Competing interests}

The author declares that they have no competing interests.

\section{Author's contributions}

The author completed the paper. The author read and approved the final manuscript.

\section{Acknowledgements}

The author would like to thank the editor and the anonymous referees for their valuable comments and helpful suggestions that improved the quality of the paper. Moreover, the author would like to thank his supervisor Professor Kefeng Liu for his constant encouragement and help. This work is supported by the Postdoctoral Science Foundation of China (2013M531342) and the Fundamental Research Funds for the Central Universities (NS2012065).

\section{Received: 31 May 2013 Accepted: 9 August 2013 Published: 27 August 2013}

\section{References}

1. Li, JY: Gradient estimates and Harnack inequalities for nonlinear parabolic and nonlinear elliptic equations on Riemannian manifolds. J. Funct. Anal. 100, 233-256 (1991)

2. Yang, YY: Gradient estimates for the equation $\Delta u+C u^{-\alpha}=0$ on Riemannian manifolds. Acta Math. Sin. 26, 1177-1182 (2010)

3. Chen, L, Chen, WY: Gradient estimates for positive smooth f-harmonic functions. Acta Math. Sci., Ser. B 30, 1614-1618 (2010)

4. Huang, GY, Li, HZ: Gradient estimates and entropy formulae of porous medium and fast diffusion equations for the Witten Laplacian. arXiv:1203.5482v1 [math.DG] (2012)

5. Li, XD: Liouville theorems for symmetric diffusion operators on complete Riemannian manifolds. J. Math. Pures Appl. 84, 1295-1361 (2005)

6. Zhang, J, Ma, BQ: Gradient estimates for a nonlinear equation $\Delta_{f} u+C u^{-\alpha}=0$ on complete noncompact manifolds. Commun. Math. 19, 73-84 (2011)

7. Zhu, XB: Hamilton's gradient estimates and Liouville theorems for fast diffusion equations on noncompact Riemannian manifolds. Proc. Am. Math. Soc. 139, 1637-1644 (2011)

8. Song, XF, Zhao, L: Gradient estimates for the elliptic and parabolic Lichnerowicz equations on compact manifolds. Z. Angew. Math. Phys. 61, 655-662 (2010)

9. Cheng, SY, Yau, ST: Differential equations on Riemannian manifolds and their geometric applications. Commun. Pure Appl. Math. 28, 333-354 (1975)

10. Aubin, T: Nonlinear Analysis on Manifolds. Springer, New York (1982) 
11. Davies, EB: Heat Kernels and Spectral Theory. Cambridge University Press, Cambridge (1989)

12. Negrin, ER: Gradient estimates and a Liouville type theorem for the Schrödinger operator. J. Funct. Anal. 127, 198-203 (1995)

doi:10.1186/1687-2770-2013-190

Cite this article as: Zhao: Harnack inequality for parabolic Lichnerowicz equations on complete noncompact Riemannian manifolds. Boundary Value Problems 2013 2013:190.

Submit your manuscript to a SpringerOpen ${ }^{\circ}$ journal and benefit from:

- Convenient online submission

- Rigorous peer review

- Immediate publication on acceptance

- Open access: articles freely available online

- High visibility within the field

- Retaining the copyright to your article

Submit your next manuscript at $\gg$ springeropen.com 